\title{
Direct-Bandgap 2D Silver-Bismuth lodide Double Perovskite: The Structure-Directing Influence of an Oligothiophene Spacer Cation
}

\author{
Manoj K. Jana, ${ }^{\dagger}$ Svenja M. Janke, ${ }^{\dagger, \|}$ David J. Dirkes, ${ }^{\dagger}$ Seyitliyev Dovletgeldi, ${ }^{\S}$ Chi Liu, ${ }^{\|}$Xixi Qin, ${ }^{\dagger}$ \\ Kenan Gundogdu, ${ }^{\S}$ Wei You, ${ }^{\ddagger}$ Volker Blum, ${ }^{\dagger, \|_{\odot}}$ and David B. Mitzi ${ }^{*}, \dagger, \|_{\odot}$ \\ ${ }^{\dagger}$ Department of Mechanical Engineering and Materials Science, Duke University, Durham, North Carolina 27708, United States \\ ${ }^{\ddagger}$ Department of Chemistry, University of North Carolina at Chapel Hill, Chapel Hill, North Carolina 27599, United States \\ ${ }^{\S}$ Department of Physics, North Carolina State University, Raleigh, North Carolina 27695, United States \\ "Department of Chemistry, Duke University, Durham, North Carolina 27708, United States
}

\begin{abstract}
Three-dimensional (3D) hybrid organic-inorganic lead halide perovskites (HOIPs) feature remarkable optoelectronic properties for solar energy conversion but suffer from longstanding issues of environmental stability and lead toxicity. Associated two-dimensional (2D) analogues are garnering increasing interest due to superior chemical stability, structural diversity, and broader property tunability. Toward lead-free 2D HOIPs, double perovskites (DPs) with mixed-valent dual metals are attractive. Translation of mixed-metal DPs to iodides, with their prospectively lower bandgaps, represents an important target

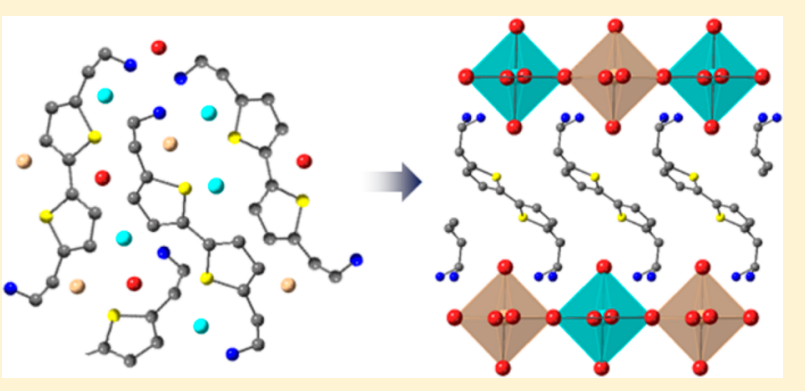
for semiconducting halide perovskites, but has so far proven inaccessible using traditional spacer cations due to either intrinsic instability or formation of competing non-perovskite phases. Here, we demonstrate the first example of a 2D Ag-Bi iodide DP with a direct bandgap of $2.00(2) \mathrm{eV}$, templated by a layer of bifunctionalized oligothiophene cations, i.e., (bis-aminoethyl)bithiophene, through a collective influence of aromatic interactions, hydrogen bonding, bidentate tethering, and structural rigidity. Hybrid density functional theory calculations for the new material reveal a direct bandgap, consistent with the experimental value, and relatively flat band edges derived principally from Ag-d/I-p (valence band) and Bi-p/I-p (conduction band) states. This work opens up new avenues for exploring specifically designed organic cations to stabilize otherwise inaccessible $2 \mathrm{D}$ HOIPs with potential applications for optoelectronics.
\end{abstract}

\section{INTRODUCTION}

Two-dimensional (2D) hybrid organic-inorganic perovskites (HOIPs) are gaining increasing attention owing to their remarkable optoelectronic/semiconducting properties and promise for applications in transistors, ${ }^{1,2}$ light-emitting diodes, ${ }^{3-5}$ photovoltaics, ${ }^{6-10}$ and spintronics. ${ }^{11,12}$ In contrast to $\left(\mathrm{CH}_{3} \mathrm{NH}_{3}\right) \mathrm{PbI}_{3}$ (the archetypal 3D HOIP), the 2D analogues exhibit superior chemical stability and are structurally more diverse, with ample choices for the organic cation. ${ }^{13,14} 2 \mathrm{D}$ HOIPs (general formula $\mathrm{A}_{2} \mathrm{MX}_{4}$ ) can be viewed as self-assembled quantum well (QW) heterostructures, ${ }^{15,16}$ where different semiconducting metal halide $\left(\mathrm{MX}_{4}^{2-}\right)$ perovskite and organic $\left(\mathrm{A}^{+}\right)$cation layers allow for at least four distinct types of internal band alignments across the organicinorganic interface. ${ }^{17,18}$ These distinct band alignments offer multiple avenues to manipulate the overall optical/electronic functionalities, ${ }^{17,19-21}$ to control carrier dynamics, ${ }^{18}$ and to further tailor the relative energy levels and optoelectronic properties by compositional and dimensionality engineering of the inorganic layers ${ }^{22}$ and/or tweaking the chemical nature of the organic cations. ${ }^{23,24}$
In the search for $\mathrm{Pb}$-free $2 \mathrm{D}$ HOIPs, it is desirable to retain the electronic structure of Pb-based HOIPs, because their direct-bandgap $\mathrm{p}-\mathrm{p}$ transitions enabled by the $\mathrm{Pb} \mathrm{s}$ lone pair allow for strong optical absorption. ${ }^{25}$ Isoelectronic cations to $\mathrm{Pb}^{\mathrm{II}}$, such as $\mathrm{Bi}^{\mathrm{II}}$, are naturally attractive candidates for exploring Pb-free HOIPs. However, $\langle 100\rangle$-oriented 2D HOIPs with layers of corner-sharing $\mathrm{M}^{\mathrm{III}} \mathrm{X}_{6}\left(\mathrm{M}=\mathrm{Bi}^{\mathrm{III}} ; \mathrm{X}=\right.$ halogen $)$ octahedra do not usually form because the $\mathrm{M}^{\mathrm{III}}$ oxidation state necessitates energetically unfavorable metal vacancies for charge balance between the organic cations and anionic metal halide layers. More commonly, $\mathrm{Bi}^{\mathrm{III}}$ forms either discrete (monomeric/dimeric) or extended (polymeric) inorganic frameworks of corner-, edge-, or face-sharing $\mathrm{MX}_{6}$ octahedra, resulting in a diverse family of halogenoanions (e.g., $\left[\mathrm{MX}_{4}\right]^{-}$, $\left[\mathrm{MX}_{5}\right]^{2-},\left[\mathrm{MX}_{6}\right]^{3-},\left[\mathrm{M}_{2} \mathrm{X}_{9}\right]^{3-},\left[\mathrm{M}_{2} \mathrm{X}_{11}\right]^{5-},\left[\mathrm{M}_{3} \mathrm{X}_{11}\right]^{2-}$ $\left[\mathrm{M}_{3} \mathrm{X}_{12}\right]^{3-},\left[\mathrm{M}_{4} \mathrm{X}_{18}\right]^{6-},\left[\mathrm{M}_{5} \mathrm{X}_{18}\right]^{3-},\left[\mathrm{M}_{6} \mathrm{X}_{22}\right]^{4-}$, and $\left[\mathrm{M}_{8} \mathrm{X}_{30}\right]^{6-}$ ) that are all characterized by a fully occupied $\mathrm{M}$ site. $^{26-29}$

Received: March 16, 2019

Published: April 23, 2019 


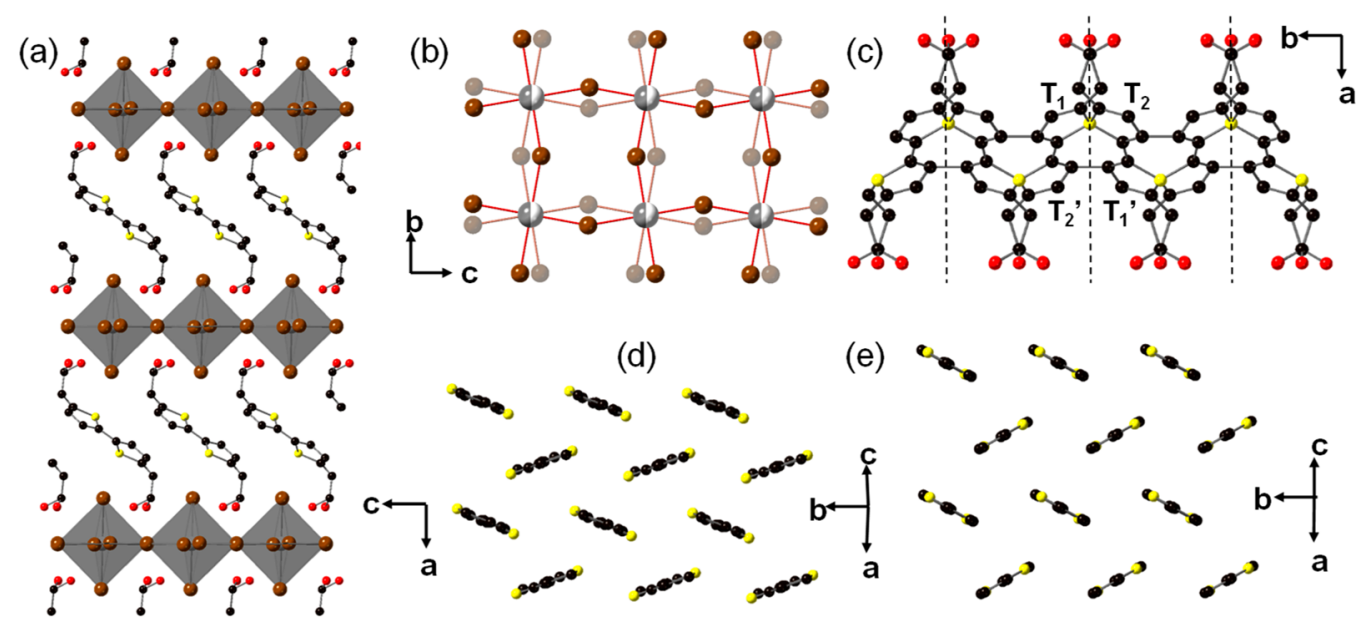

Figure 1. (a) Schematic showing the $2 \mathrm{D}$ layered crystal structure $\left(\mathrm{C} 2 / \mathrm{c}\right.$ space group) of the $[\mathrm{AE} 2 \mathrm{~T}]_{2} \mathrm{AgBiI}{ }_{8} \mathrm{DP}$. (b) Two distorted frameworks of interconnected equatorial $\mathrm{M}-\mathrm{I}_{\mathrm{eq}}-\mathrm{M}$ bonds superimpose to give rise to two sets of half-occupied equatorial iodine atoms (denoted by solid and shaded brown spheres). Randomly occupied Ag/Bi sites are shown as two-colored spheres. (c) View of AE2T cations disordered across the $c$-glide plane (indicated as dotted lines), thereby appearing as polymeric ribbons along the $b$-axis. Considering only one set of disordered bithiophene moieties, the herringbone packing arrangement in a single organic layer of $(\mathrm{d})[\mathrm{AE} 2 \mathrm{~T}]_{2} \mathrm{AgBiI}_{8}$ closely matches that in (e) "neat" $2,2^{\prime}$-bithiophene crystals along a similar viewing direction. Ethylammonium groups are omitted in (d) for clarity. Black, red, and yellow spheres denote $\mathrm{C}$, N, and S atoms, respectively.

Another way of meeting the valence requirements is to incorporate two metals with distinct oxidation states. ${ }^{30-36}$ To form double perovskites (DPs), both the metal sites additionally need to adopt stable octahedral coordination with a given halide ion. Various inorganic 3D DPs (e.g., $\mathrm{Cs}_{2} \mathrm{M}^{\mathrm{I}} \mathrm{M}^{\mathrm{III}} \mathrm{X}_{6} ; \mathrm{X}=$ $\mathrm{Cl}, \mathrm{Br}$ ) containing a trivalent cation $\left(\mathrm{M}^{\mathrm{III}}\right)$ such as $\mathrm{Bi}, \mathrm{Sb}$, In, or $\mathrm{Tl}$ and a monovalent cation $\left(\mathrm{M}^{\mathrm{I}}\right)$ such as $\mathrm{Ag}$ or $\mathrm{Tl}$ have been reported in the literature. ${ }^{30-34}$ Most known 3D DPs of this family are characterized by large indirect bandgaps, while only a few possess direct bandgaps, e.g., $\mathrm{Cs}_{2} \mathrm{AgInCl}_{6}{ }^{30}$ and $\mathrm{Cs}_{2} \mathrm{AgTlX}_{6}(\mathrm{X}=\mathrm{Cl}, \mathrm{Br}) .{ }^{34}$ Although predicted to exhibit direct bandgaps, $\mathrm{In}(\mathrm{I})$-based DPs such as $\mathrm{Cs}_{2} \mathrm{In}^{\mathrm{I}} \mathrm{M}^{\mathrm{III}} \mathrm{X}_{6}\left(\mathrm{M}^{\mathrm{III}}=\right.$ $\mathrm{Bi} / \mathrm{Sb} ; \mathrm{X}=\mathrm{Cl}, \mathrm{Br}, \mathrm{I})$ are intrinsically unstable against oxidation into In(III)-based binary compounds. ${ }^{37}$ Due to their expected lower bandgaps, the iodide analogues are of special interest for semiconductors in optoelectronic applications. So far, however, this family of 3D DPs could not be extended to iodides with any of the above metal combinations; ${ }^{36} \mathrm{Cs}_{2} \mathrm{AgBiI}_{6}$ could only be kinetically stabilized in colloidal form by a postsynthetic anion exchange from the corresponding chlorides and bromides, ${ }^{38}$ but the bulk counterpart has not been formed.

Two-dimensional hybrid DPs may open a new avenue for stabilizing iodide-based DPs due to a number of possible choices of potentially stabilizing organic spacer cations. However, while a $2 \mathrm{D}$ DP bromide, $(\mathrm{BA})_{4} \mathrm{AgBiBr}_{8}(\mathrm{BA}=$ $\left.\mathrm{CH}_{3}\left(\mathrm{CH}_{2}\right)_{3} \mathrm{NH}_{3}^{+}\right)$, with ordered $\mathrm{Ag}$ and $\mathrm{Bi}$ sites and electronically inactive $\mathrm{BA}^{+}$cations was recently reported, ${ }^{39}$ the electronically desirable 2D mixed-metal iodide DPs have not yet been reported (to our knowledge, the only iodide DPs so far reported are based on mixed-valent homometal $\mathrm{Au}(\mathrm{I}) /$ $\mathrm{Au}(\mathrm{III})$ iodides $\left.^{40,41}\right)$. Strikingly, we find that their stabilization is not just a matter of changing the halide anion. In our own synthesis attempts of iodide-based $\mathrm{Ag}-\mathrm{Bi} 2 \mathrm{D}$ DPs with $\mathrm{BA}^{+}$ and related cations, competing $\mathrm{OD}$ or $1 \mathrm{D}$ frameworks form instead (see below, as well as "Experimental Methods" and Figure S4 in the Supporting Information, SI). This is due to an intrinsic propensity of $\mathrm{Bi}$ to form low-dimensional nonperovskite hybrids, aggravated by the rareness of $\mathrm{Ag}$ in octahedral iodine coordination.
Here, we demonstrate the structure-directing influence of a specifically designed bifunctionalized organic spacer molecule-5,5'-diylbis(aminoethyl)-[2,2'-bithiophene] (AE2T)which, as a result of preferential edge-to-face aromatic interactions, acts as a template to stabilize the first example of a lead-free $2 \mathrm{D}$ mixed-metal DP iodide, i.e., $[\mathrm{AE} 2 \mathrm{~T}]_{2} \mathrm{AgBiI}_{8}$. Our work thus proves the value of employing specifically designed templating organic cation layers to stabilize unusual $2 \mathrm{D}$ perovskite phases that are often inaccessible using traditional spacer cations. Using spin-orbit coupled hybrid density functional theory (DFT), we also find that, in contrast to traditional spacer cations, in $[\mathrm{AE} 2 \mathrm{~T}]_{2} \mathrm{AgBiI}_{8}$, the frontier orbitals of AE2T are in close proximity to the inorganic band edges, resulting in a borderline type-I/II quantum well alignment, while the inorganic-derived bands are much less dispersive compared to related oligothiophene- $\mathrm{Pb}$-based $2 \mathrm{D}$ HOIPs. ${ }^{18}$ Experimentally, the optical transition in $[\mathrm{AE} 2 \mathrm{~T}]_{2^{-}}$ $\mathrm{AgBiI}_{8}$ is weaker and broader compared to the sharp excitonic resonance commonly found in Pb-based HOIPs ${ }^{42}$ and can be associated with a transition between equatorial I-p states in the valence band maximum (VBM) and hybridized equatorial I-p and Bi-p orbitals in the conduction band minimum (CBM).

\section{RESULTS AND DISCUSSION}

Structural Characteristics. Single crystals of $[\mathrm{AE} 2 \mathrm{~T}]_{2}$ $\mathrm{AgBiI}_{8}$ were grown by slowly cooling a saturated aqueous $\mathrm{HI}$ solution of stoichiometric amounts of $\mathrm{AgI}, \mathrm{BiI}_{3}$, and AE2T.2HI from $100{ }^{\circ} \mathrm{C}$ over $60 \mathrm{~h}$ (see "Experimental Methods" in the SI for more details). AE2T.2HI was synthesized starting from thiophene-2-ethylamine (see Scheme S1 and detailed description in the SI).

$[\mathrm{AE} 2 \mathrm{~T}]_{2} \mathrm{AgBiI}_{8}$ crystallizes into a $\langle 100\rangle$-oriented perovskite, where each negatively charged inorganic perovskite layer comprises corner-sharing $\mathrm{MI}_{6}[\mathrm{M}=\mathrm{Ag}$ or Bi] octahedra, with adjacent layers separated by layers of AE2T cations (Figure 1a). For $[\mathrm{AE} 2 \mathrm{~T}]_{2} \mathrm{AgBiI}{ }_{8}$ crystals, two sets of reflections were observed from the CCD frames during the X-ray study at 298 $\mathrm{K}$ : one with a well-defined, relatively sharp profile and the other with diffuse, streak-like character. The former corre- 
sponds to a monoclinic subcell ( $C 2 / c$ space group) with lattice parameters $a=29.3654(14) \AA, b=6.1473(3) \AA, c=$ 12.0494(6) $\AA$, and $\beta=92.732(1)^{\circ}$, whereas the latter corresponds to a poorly resolved superstructure resulting in doubled $b$ - and/or $a$-axes. The precession image of the reciprocal lattice reconstructed along the $h k 0$ section (Figure S1) also reveals poorly resolved reflections forming streaks along the $a^{*}$-direction, plausibly due to a weak superstructure related to stacking of adjacent layers. ${ }^{17,43,44}$ The adjacent $\mathrm{MI}_{6}$ $(\mathrm{M}=\mathrm{Ag} / \mathrm{Bi})$ octahedra in each inorganic layer are twisted with respect to each other, so that $\mathrm{M}-\mathrm{I}-\mathrm{M}$ bond angles substantially deviate from $180^{\circ}\left[160.50(5)^{\circ}\right.$ and $\left.159.43(5)^{\circ}\right]$. Within a given inorganic layer, any given square of four adjacent corner-sharing $\mathrm{MI}_{6}$ octahedra (defined by four interconnected $\mathrm{M}-\mathrm{I}-\mathrm{M}$ bonds) is "pulled-in" and "pushedout" along the $b$ - and $c$-axes, respectively, or vice versa. The distortion pattern of each square fixes the nearest-neighboring square of octahedra to be in the opposite pattern (Figure $1 \mathrm{~b}$ ). The distortions in each inorganic layer $(b-c$ plane) are, therefore, fully ordered; however, the adjacent layers are not in registry with each other. Such diffuse scattering arising from a poorly ordered superstructure is not uncommon among layered 2D HOIPs, having also been observed earlier in (3fluorophenethylammonium) ${ }_{2} \mathrm{SnI}_{4}{ }^{43}$ (2-fluorophenethylammonium $)_{2} \mathrm{SnI}_{4}{ }^{43}$ (AE4T) $\mathrm{PbBr}_{4}$, ${ }^{17}$ and $[\mathrm{AE} 4 \mathrm{~T}] \mathrm{Bi}_{2 / 3} \square_{1 / 3} \mathrm{I}_{4},{ }^{44}$ whose structures have been refined using a similar subcell approach. The $[\mathrm{AE} 2 \mathrm{~T}]_{2} \mathrm{AgBiI}_{8}$ crystal structure can be solved adequately using the $C 2 / c$ subcell (see Table $S 1$ in the SI), but no meaningful structure solution could be obtained using the larger supercell approach, likely due to the poorly resolved Xray peaks (and ordering) corresponding to the larger cell.

Within the inorganic layer of $[\mathrm{AE} 2 \mathrm{~T}]_{2} \mathrm{AgBiI}_{8}, \mathrm{Ag}$ and $\mathrm{Bi}$ cations do not appear to exhibit long-range order on the $M$ site and are instead successfully modeled as being statistically distributed, where the chemical occupancy of $\mathrm{Ag}$ and Bi refines to $\sim 0.5$ for each. Consistently, both energy-dispersive X-ray spectroscopy (EDS) and X-ray photoelectron spectroscopy (XPS) quantitative analyses on single crystals confirm nearly equal proportions of $\mathrm{Ag}$ and $\mathrm{Bi}$ (Figures S2 and S3, SI). We note that the apparent absence of long-range order in diffraction does not rule out the existence of local order (or order within a given layer, as opposed to registry between the layers), as indicated by our DFT results below. Each equatorial iodine in the inorganic layer is disordered over two symmetryrelated sites with an occupancy of 0.5 . The disorder of equatorial iodine atoms is a consequence of the poorly resolved superstructure associated with the lack of registry between adjacent inorganic layers. In the subcell model, the two unique patterns of square distortion are superimposed with equal probability, resulting in an apparent disorder of equatorial iodine atoms (Figure $1 \mathrm{~b}$ ). The lack of registry between adjacent inorganic layers is plausible given that the ethylammonium tethering groups of the AE2T cations, nominally interacting with the distorted square of $\mathrm{MI}_{6}$ octahedra through hydrogen bonding with nearest iodine atoms, are flexible enough to adopt different conformations corresponding to the two different square distortions.

In the organic layer, all the carbon atoms (except the terminal one closest to the nitrogen) and nitrogen atoms of the $\mathrm{AE} 2 \mathrm{~T}$ cation are disordered across the c-glide plane (Figure 1c). The asymmetric unit contains two unique thiophene rings ( $\mathrm{T}_{1}$ and $\mathrm{T}_{2}$ ), and the other half of each bithiophene moiety (i.e., $\mathrm{T}_{1}{ }^{\prime}$ and $\mathrm{T}_{2}{ }^{\prime}$ ) is generated by $2_{1}$ symmetry. The two thiophene rings within each AE2T moiety are nearly coplanar with a dihedral angle of $0.66^{\circ}$ between least-squares best planes containing the individual thiophene rings. The adjacent thiophene rings adopt an "anti" configuration (see Figure 1c), as typically found in neat organic crystals of unsubstituted bithiophene molecules ${ }^{45}$ as well as related hybrids incorporating substituted bithiophene cations. ${ }^{46}$ The disorder for the AE2T cation is also consistent with the poorly defined/ resolved superstructure; that is, the diffuse scattering along $a^{*}$ doubles the $b$-axis, but in the subcell $\left(\sim 1_{\mathrm{b}} \times 2_{\mathrm{c}}\right)$ model considered here, the two half-occupied (i.e., equal probability) configurations of the AE2T backbone become superimposed to extend as ribbons along the $b$-direction (Figure 1c). Similar disordering of the organic cation across symmetry elements was found in $[\mathrm{AE} 4 \mathrm{~T}] \mathrm{Bi}_{2 / 3} \square_{1 / 3} \mathrm{I}_{4}$, which also exhibits a poorly resolved superstructure. ${ }^{44}$

$\mathrm{X}$-ray data collected at $100 \mathrm{~K}$ for $[\mathrm{AE} 2 \mathrm{~T}]_{2} \mathrm{AgBiI}_{8}$ also exhibits a diffuse scattering due to the poorly resolved superstructure. The $2 \mathrm{D}$ perovskite structure at $100 \mathrm{~K}$ can be solved adequately in a monoclinic $C 2 / c$ subcell with lattice parameters $a=28.951(3) \AA, b=6.0992(6) \AA ̊, c=11.9821$ (12) $\AA$, and $\beta=92.266(2)^{\circ}$ (see Table S1 in the SI) and features disordered inorganic and organic layers similar to the $298 \mathrm{~K}$ structure.

Templating Influence of Oligothiophene Spacer Cations. In 2000, Mitzi reported the unique templating influence of an oligothiophene-based diammonium cation in stabilizing an unusual Bi-deficient $\langle 100\rangle$-oriented 2D HOIP, [AE4T] $\mathrm{Bi}_{2 / 3} \square_{1 / 3} \mathrm{I}_{4}$ (AE4T: 5,5 '" -bis (aminoethyl)$2,2^{\prime}: 5^{\prime}, 2^{\prime \prime}: 5^{\prime \prime}, 2^{\prime \prime \prime}$-quaterthiophene). ${ }^{44}$ The latter HOIP features $2 \mathrm{D}$ layers of corner-sharing distorted $\mathrm{BiI}_{6}$ octahedra with randomly distributed $\mathrm{Bi}$-vacancies and represents the first member of a new family of metal-deficient 2D HOIPs with $\left[\mathrm{M}_{2 / n}^{n+} \square_{(n-2) / n} \mathrm{X}_{4}\right]^{2-}$ layers $(\square=$ M-site vacancy). The templating role of the AE4T cation was ascribed to edge-toface (tilted-T type) intermolecular aromatic interactions that are strong enough to compensate for an otherwise unfavorable formation enthalpy of a substantial density of Bi-vacancies (i.e., $1 / 3$ of the sites) within the perovskite layer. Notably, another complex but relatively flexible diammonium organic cation, [1,6-bis [ $5^{\prime}-\left(2^{\prime \prime}\right.$-aminoethyl $)-2^{\prime}$-thienyl $]$ hexane $]$, wherein the two terminal thienyl rings are separated by a hexyl chain (as opposed to a bithiophene unit in AE4T), leads to a onedimensional (1D) hybrid with corner-sharing zigzag chains of distorted $\mathrm{BiI}_{6}$ octahedra, ${ }^{47}$ emphasizing the importance of structural rigidity in addition to the aromatic interactions for stabilizing these more complex metal-deficient $2 \mathrm{D}$ perovskites.

As the number of thiophene rings within the oligothiophene moiety is systematically decreased from four (AE4T) to three (AE3T) and down to two (AE2T), the strength of aromatic interactions is expected to decrease, thereby influencing the overall templating ability. For instance, free-energy calculations show a decreasing gas-phase interaction enthalpy going from anthracene to naphthalene to benzene dimers, accompanied by a change in preference for $\pi$-stacked structure in anthracene dimers to tilted-T-type structure in benzene dimers. ${ }^{48}$ In the current study, we focus on the 2D DP with a 1:1 mixture of Bi and $\mathrm{Ag}$ atoms, to achieve an average +2 oxidation state for the metal site without vacancies. Such structures are still subject to instability due to the availability of more stable alternative phases (i.e., those with $0 \mathrm{D}$ or $1 \mathrm{D} \mathrm{Bi}-\mathrm{I}$ frameworks). To stabilize the $2 \mathrm{D}$ structure, we have employed the smaller 
(relative to AE4T) but still rigid AE2T cation and find that the aromatic interactions in AE2T are sufficiently strong to template the targeted lead-free 2D DP iodide, i.e., $[\mathrm{AE} 2 \mathrm{~T}]_{2} \mathrm{AgBiI}_{8}$. AE2T cations were selected here (as opposed to $\mathrm{AE} 4 \mathrm{~T}$ ) because they are more readily employed (i.e., exhibit higher solubility) for synthesizing the hybrid 2D DP.

The bithiophene moieties within a single organic layer of $[\mathrm{AE} 2 \mathrm{~T}]_{2} \mathrm{AgBiI}_{8}$ are arranged in a herringbone pattern (Figure 1d) with edge-to-face (tilted-T type) aromatic interactions, similar to that observed in $[\mathrm{AE} 4 \mathrm{~T}] \mathrm{Bi}_{2 / 3} \square_{1 / 3} \mathrm{I}_{4}$ and in the "neat" 2,2'-bithiophene organic crystals (Figure 1e). ${ }^{45}$ The herringbone angle between the least-squares best planes through the adjacent AE2T cations (excluding the terminal ethylammonium tethering units) in $[\mathrm{AE} 2 \mathrm{~T}]_{2} \mathrm{AgBiI}_{8}$ is $42.97^{\circ}$, which can be compared to the $58.35^{\circ}$ value in bithiophene crystals. Despite being in a very different chemical environment (i.e., in between metal halide layers), the preferred herringbone arrangement of bithiophene units within 2D layers, favored by the edge-to-face interactions, is able to template the formation of $2 \mathrm{D}$ metal halide layers in $[\mathrm{AE} 2 \mathrm{~T}]_{2} \mathrm{AgBiI}_{8}$. In a gas-phase benzene dimer, the stable aromatic interaction is also of the tilted- $T$ type, with interaction energy of about $-2 \mathrm{kcal} / \mathrm{mol}^{48}$ which is comparable to a weak hydrogen bond. A similar order of magnitude for the interaction energy can be expected for the tilted-T-type interactions of neighboring AE2T cations in $[\mathrm{AE} 2 \mathrm{~T}]_{2} \mathrm{AgBiI}_{8}$.

Notably, we found that a linear $n$-butylammonium (BA) cation, as used in $(\mathrm{BA})_{4} \mathrm{AgBiBr}_{8} \mathrm{DP},{ }^{39}$ or its diammonium analogue, butane-1,4-diammonium (BDA), could not stabilize a $2 \mathrm{D}$ DP iodide under synthesis conditions similar to those used to form $[\mathrm{AE} 2 \mathrm{~T}]_{2} \mathrm{AgBiI}_{8}$. Instead, a hybrid $\mathrm{Bi}$ iodide with more typical $0 \mathrm{D}$ (or $1 \mathrm{D}$ ) connectivity crystallizes from the solution containing stoichiometric amounts of $\mathrm{AgI}, \mathrm{BiI}_{3}$, and BA (or BDA) (see Experimental Methods and Figure S4 in the SI for more details). Moreover, an asymmetrical, monosubstituted bithiophene cation with a sulfur ethylammonium tethering group (AESBT: $\left.\left(\mathrm{C}_{4} \mathrm{~S}\right)_{2} \mathrm{~S}\left(\mathrm{CH}_{2}\right)_{2} \mathrm{NH}^{3+}\right)$ was previously shown to form non-perovskite hybrids with $\mathrm{Pb}$ and $\mathrm{Bi}$, namely, (AESBT) ${ }_{4} \mathrm{~Pb}_{3} \mathrm{I}_{10}$ and (AESBT) ${ }_{3} \mathrm{Bi}_{2} \mathrm{I}_{9}$. ${ }^{46}$ $(\mathrm{AESBT})_{4} \mathrm{~Pb}_{3} \mathrm{I}_{10}$ exhibits a herringbone molecular packing of organic cations, while (AESBT) ${ }_{3} \mathrm{Bi}_{2} \mathrm{I}_{9}$ exhibits a mixed packing with alternating herringbone and k-type arrangements. ${ }^{46}$ Therefore, in addition to the associated aromatic interactions and the hydrogen-bonding interactions of ammonium tethering groups, mono- vs bisubstitution of the spacer cation impacts the stability of the $2 \mathrm{D}$ perovskite structure. These observations emphasize that multiple aspects of the AE2T cation synergistically converge to template an otherwise unstable 2D DP iodide, i.e., strong intermolecular edge-toface aromatic interactions that intrinsically drive AE2T cations to pack into $2 \mathrm{D}$ herringbone layers, structural rigidity of the AE2T backbone, and tethering ammonium groups on either end of the bithiophene moiety that drive self-assembly of $2 \mathrm{D}$ DP layers via hydrogen-bonding interactions with the latter.

It is further noted that both the metal sites in a DP halide must adopt stable octahedral coordination with the halide. Bi typically exhibits octahedral coordination with $\mathrm{I}$, whereas $\mathrm{Ag}$ by itself rarely exhibits octahedral iodine coordination, but rather usually prefers tetrahedral coordination. In contrast, sixcoordinated $\mathrm{Ag}$ is found often in bromides, the simplest example being $\mathrm{AgBr}$ with a rock-salt structure. However, Bi can occasionally facilitate octahedral coordination of $\mathrm{Ag}$ with I as found in $\mathrm{AgBiI}_{4}$ and $\mathrm{Ag}_{3} \mathrm{BiI}_{6}$, where $\mathrm{Ag}$ and $\mathrm{Bi}$ exhibit site disorder. ${ }^{49}$ This site mixing, in conjunction with the templating influence from AE2T, helps to stabilize the unprecedented iodide-based 2D DP structure found for $[\mathrm{AE} 2 \mathrm{~T}]_{2} \mathrm{AgBiI}_{8}$.

First-Principles Calculations. To understand the electronic structure of the $\mathrm{Ag}-\mathrm{Bi} 2 \mathrm{D}$ perovskite framework, we carried out first-principles DFT calculations within the allelectron electronic structure code FHI-aims. ${ }^{50-54}$ We first optimized geometries for $[\mathrm{AE} 2 \mathrm{~T}]_{2} \mathrm{AgBiI}_{8}$ until all residual forces on the nuclei were below $0.005 \mathrm{eV} / \AA$ in magnitude, based on the experimental structure and using the PBE exchange-correlation functional ${ }^{55}$ plus the TkatchenkoScheffler (TS) pairwise dispersion scheme, ${ }^{56}$ "tight" basis sets, and other numerical defaults within FHI-aims. The electronic structure of the perovskite is then investigated based on the DFT-PBE+TS relaxed geometries using the established HSE06 hybrid density functional with fixed values $\alpha=0.25$ and $\omega=0.11 \mathrm{bohr}^{-1}$ for the exchange mixing parameter and range separation parameter, respectively. ${ }^{57-59}$ The accuracy of this set of approaches for oligothiophene-based 2D perovskites was demonstrated by extensive validation in ref 18 and its SI. Spin-orbit coupling (SOC) is treated using a secondvariational, non-self-consistent approach, rendering the expensive but necessary hybrid DFT band structure calculations computationally feasible. ${ }^{60}$ In ref 60 , a broad analysis of different computational approaches to SOC shows that the non-self-consistent approach remains qualitatively accurate even for $\mathrm{Bi}$, but quantitative differences to a self-consistent SOC treatment become significant (about $10 \%$ of the overall SOC splitting for the Bi-6p-derived states). In Figures S5 and S6 (SI), we therefore assess the accuracy of the SOC treatment for $\mathrm{Bi}-6 \mathrm{p}$-derived conduction band states specifically for a simpler Ag-Bi-based DP, $\mathrm{Cs}_{2} \mathrm{AgBiCl}_{6},{ }^{61}$ comparing to a fully self-consistent implementation including $\mathrm{p}_{1 / 2}$ local orbitals in the basis set, as implemented in the all-electron augmented plane wave code WIEN2K. ${ }^{62}$ This analysis reveals a relative accuracy of the Bi-6p-derived states of about $0.4 \mathrm{eV}$, similar to the expected quality of the HSE06 density functional ${ }^{63-65}$ and supporting our overall approach.

In order to accurately model the octahedral distortion pattern in the inorganic layers (Figure $1 b)$, a $(2 \times 2)$ supercell is required (as in the case of oligothiophene-containing $\mathrm{Pb}$-based perovskites $\left.^{18}\right)$. Within the $(2 \times 2)$ supercell, we chose a checkerboard-like $\mathrm{Ag}-\mathrm{Bi}$ pattern (see Figure S7a and c). We note that different $\mathrm{Ag}-\mathrm{Bi}$ patterns are possible in principle. The experimental XRD pattern does not clarify this point since the site disorder assumed in the refinement can result as a consequence of lack of registry between different $\mathrm{Ag}-\mathrm{Bi}$ planes. Within the supercell, only one other $\mathrm{Ag}-\mathrm{Bi}$ arrangement is possible, consisting of adjacent rows of $\mathrm{Ag}$ - and $\mathrm{Bi}$ containing iodide octahedra (Figure S7b and d). As shown in the SI, our calculations indicate significantly greater structural and energetic stability for the checkerboard-like pattern, i.e., a significant tendency of $\mathrm{Ag}$ and $\mathrm{Bi}$ to form a locally well-ordered arrangement. This is consistent with several literature reports for 3D DPs, ${ }^{66-68}$ all of which indicate high ordering energies. In ref 68 a first-principles cluster expansion of the $3 \mathrm{D}$ compound $\mathrm{Cs}_{2} \mathrm{AgBiBr}_{6}$ shows that the onset of meaningful $\mathrm{Ag}-\mathrm{Bi}$ disorder occurs only above $\sim 1000 \mathrm{~K}$, significantly higher than the expected overall stability range of the $2 \mathrm{D} D P$ investigated here. Both our own results and the literature therefore point toward the checkerboard-like $\mathrm{Ag}-\mathrm{Bi}$ arrangement within a single plane as the dominant structural motif to 

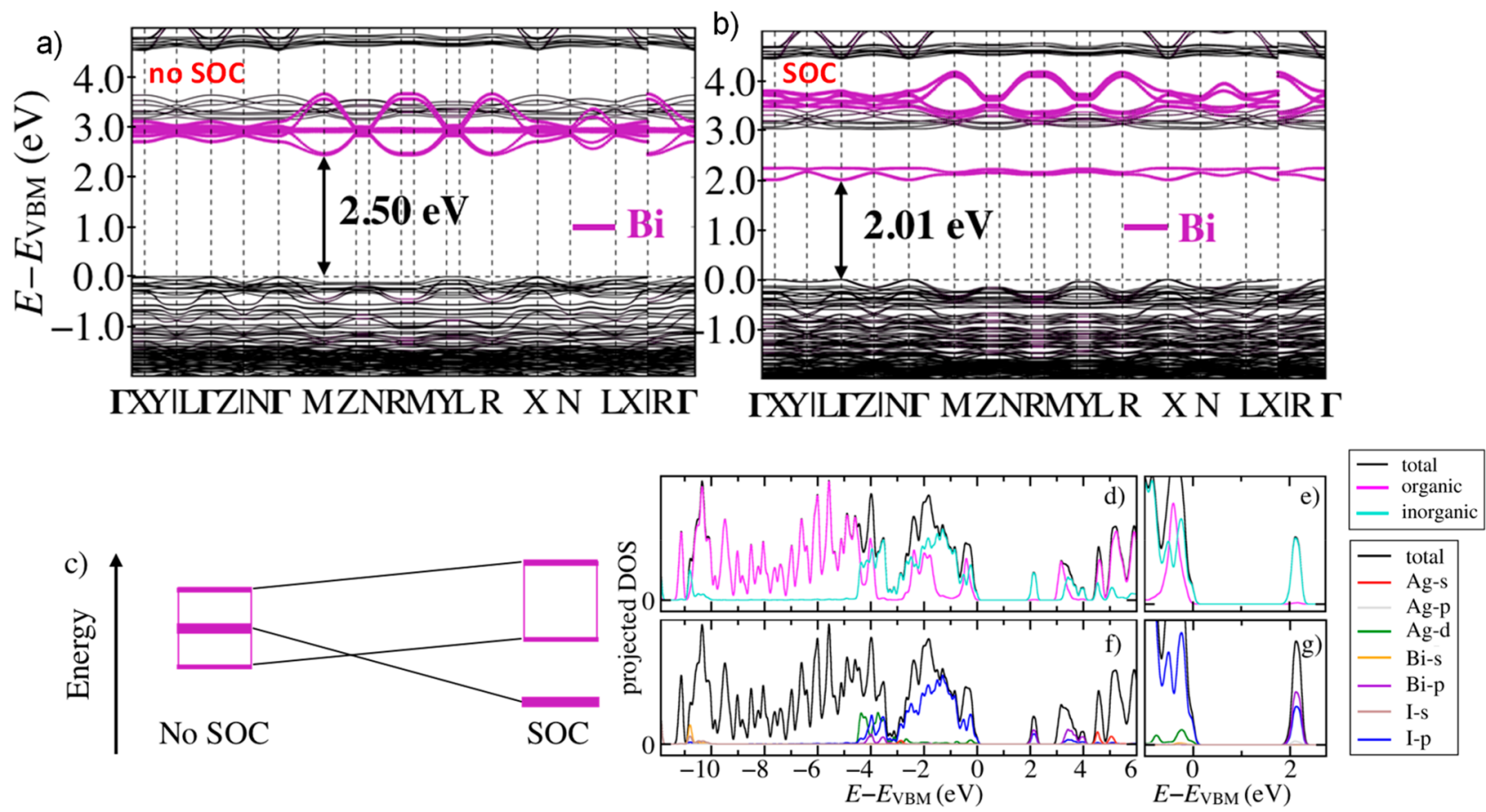

Figure 2. DFT-HSE06 band structure of $[\mathrm{AE} 2 \mathrm{~T}]_{2} \mathrm{AgBiI}_{8}$ (a) without and (b) with spin-orbit coupling (SOC). The contributions of the Bi-6p orbitals are identified (purple), and the zero in energy is set to the VBM. (c) Schematic molecular orbital diagram for the splitting of the Bi-6p orbitals into $\mathrm{p}_{1 / 2}$ and $\mathrm{p}_{3 / 2}$ contributions under SOC. (d) DFT-HSE06+SOC total density of states (DOS, black) and partial DOS projected onto the atoms of the organic compound (magenta) and the inorganic compound (turquoise). (e) Magnified region around band edges from (d). (f) Total DOS (black) and Ag-s (red), Ag-p (gray), Ag-d (green), Bi-s (orange), Bi-p (purple), I-s (brown), and I-p (blue) partial DOS. (g) Magnified region around band edges from $(\mathrm{f})$.

be considered. After structure relaxation, the predicted lattice parameters of this model agree with the experimental ones (at $100 \mathrm{~K}$ ) to within $0.4 \%$ for the $b$ and $c$ lattice parameters and to within $2.7 \%$ for the $a$ lattice parameter (see Table S3). Each metal atom has one long and one short axial bond. Long and short $\mathrm{Bi}-\mathrm{I}_{\mathrm{ax}}$ bond lengths in the supercell average to 3.29 and $3.05 \AA$, respectively, whereas long and short $\mathrm{Ag}-\mathrm{I}_{\mathrm{ax}}$ bond lengths average to 3.20 and $2.89 \AA$, respectively. Furthermore, the orientation of the short vs long axial bonds (upward or downward relative to the inorganic plane) alternates among sites within the inorganic layer. The $\mathrm{Bi}-\mathrm{I}$ bond lengths found here are typical of those encountered in bismuth iodide hybrids. $^{44,46}$ In contrast, $\mathrm{Ag}$ rarely exhibits octahedral coordination with $\mathrm{I}$, a few examples being $\mathrm{Tl}_{2} \mathrm{AgI}_{3}$ ( $\mathrm{Ag}-\mathrm{I}$ : $3.126 \AA$ ) with a regular coordination ${ }^{69}$ and $\mathrm{Ag}_{4} \mathrm{I}_{2} \mathrm{SeO}_{4}(\mathrm{Ag}-\mathrm{I}$ : 2.878-3.458 $\AA$ ) with a distorted coordination with $\mathrm{O}$ and $\mathrm{I}^{70}$ These Ag-I bond lengths are comparable to those found in our relaxed structure.

In the experimental subcell model considered here, axial MI bonds refine to an average value of 3.0705(8) $\AA$, due to the assumed random distribution of $\mathrm{Ag}$ and $\mathrm{Bi}$. The axial $\mathrm{I}$ in the 298 K structure can, however, be successfully split during structural refinement into four distinguishable sites with a fixed chemical occupancy of 0.25 for each, which may be assigned to the axial bond types, $\mathrm{Bi}-\mathrm{I}_{\mathrm{ax}}(3.334(17)$ and $3.075(17) \AA)$ and $\mathrm{Ag}-\mathrm{I}_{\mathrm{ax}}(3.080(17)$ and $2.848(5) \AA$ ) (Figure S8), in good qualitative agreement with the computationally derived values. Other predicted structural details are also in close agreement with the experimental structure (see Figures S8 and S9 for details). Comparing to the $(\mathrm{BA})_{4} \mathrm{AgBiBr}_{8} \mathrm{DP},{ }^{39}$ very short axial $\mathrm{Ag}-\mathrm{Br}$ bonds were found, but asymmetric bond lengths for $\mathrm{Bi}-\mathrm{Br}_{\mathrm{ax}}$ and $\mathrm{Ag}-\mathrm{Br}_{\mathrm{ax}}$ were only found for its highdimensional analogue with mixed $\mathrm{BA}$ and Cs cations.

Figure $2 \mathrm{a}$ and $\mathrm{b}$ show the HSE06 band structure of $[\mathrm{AE} 2 \mathrm{~T}]_{2} \mathrm{AgBiI}_{8}$. Without the inclusion of spin-orbit coupling (Figure $2 \mathrm{a})$, the predicted bandgap is indirect $(2.5 \mathrm{eV})$. The necessity to include SOC for a correct description of $\mathrm{Pb}$ - and Bi-based HOIPs is well established. ${ }^{18,71-73}$ Accordingly, and in qualitative agreement with observations for $\mathrm{Cs}_{2} \mathrm{AgBiBr}_{6}$ and $\mathrm{Cs}_{2} \mathrm{AgBiCl}_{6},{ }^{61,71}$ inclusion of SOC (Figure $2 \mathrm{~b}$ and $\mathrm{c}$ ) leads to a separation within the $\mathrm{Bi}-6 \mathrm{p}$-derived conduction band, resulting in a split-off conduction band. Consistent with analogous assignments for $3 \mathrm{D} \mathrm{DPs},{ }^{71}$ we attribute the split-off conduction band, which shifts down, to Bi- $6 \mathrm{p}_{1 / 2}$-derived states. The more disperse bands, which shift upward, are attributed to $\mathrm{Bi}-6 \mathrm{p}_{3 / 2^{-}}$ derived states. As a result of the shift, the conduction band minimum with SOC is found at the $\Gamma$ point and the bandgap including $\operatorname{SOC}(2.01 \mathrm{eV})$ is direct, in contrast to the case without SOC. Both the CBM and the VBM are isoenergetic to within $10 \mathrm{meV}$ between $\mathrm{X}(\pi / \mathrm{a}, 0,0)$ and $\Gamma(0,0,0)$. The presence of the CBM and VBM at $\Gamma$ is likely the result of band back-folding, as seen in the example of a model unit cell in the SI (Figure S10). Connor et al. ${ }^{39}$ showed that the direct bandgap in $2 \mathrm{D}(\mathrm{BA})_{4} \mathrm{AgBiBr}_{8} \mathrm{DP}$, compared to the indirect one found in higher-dimensional (i.e., $n=2$ and $n=\infty$ ) AgBibased bromide DPs, is the result of dimensionality reduction. ${ }^{39}$ Replacing $\mathrm{Pb}$ by $\mathrm{Ag}-\mathrm{Bi}$ in $\mathrm{Cs}_{2} \mathrm{~Pb}_{2} \mathrm{Br}_{6}$ has been observed to lead to less dispersive bands in the conduction band of the $\mathrm{Ag}-\mathrm{Bi}$ compound due to confinement of the frontier orbitals to either $\mathrm{Ag}(\mathrm{VBM})$ or $\mathrm{Bi}(\mathrm{CBM})$, with only second-nearestneighbor cation interactions compared to nearest-neighbor interactions in Pb-based compounds. ${ }^{67}$ In agreement with this observation, the bands in the conduction band of $[\mathrm{AE} 2 \mathrm{~T}]_{2}-$ 
$\mathrm{AgBiI}_{8}$ are relatively nondispersive (i.e., "flat") compared to the band structures of the direct-bandgap oligothiophene-containing Pb-based HOIPs, ${ }^{18}$ i.e., flat enough that the conduction band becomes isolated.

To understand the character of the frontier orbitals, we further investigated the DOS projected onto the organic and inorganic frameworks of $[\mathrm{AE} 2 \mathrm{~T}]_{2} \mathrm{AgBiI}_{8}$ (Figure $2 \mathrm{~b}$ and $\mathrm{c}$ ). The VBM and CBM are formed by the inorganic layers, but with the organic HOMO almost degenerate with, i.e., $\sim 0.03$ $\mathrm{eV}$ below, the inorganic $\mathrm{VBM}$ in $[\mathrm{AE} 2 \mathrm{~T}]_{2} \mathrm{AgBiI}_{8}$. Nominally, this alignment represents a type $\mathrm{Ib}$ heterojunction (in the notation of ref 18); however, given the remaining accuracy limits of hybrid DFT calculations, it cannot be distinguished from a type IIb heterojunction. Apart from the flatter inorganic-derived bands, the electronic structure of $[\mathrm{AE} 2 \mathrm{~T}]_{2}-$ $\mathrm{AgBiI}_{8}$ is hence similar to the electronic structure exhibited by or expected from the oligothiophene-Pb-based HOIPs. ${ }^{18}$ Figure $2 \mathrm{~d}$ and e show contributions to the inorganic frontier orbitals of $[\mathrm{AE} 2 \mathrm{~T}]_{2} \mathrm{AgBiI}_{8}$ resolved according to element and orbital character. The VBM is dominated by I-p states, with contributions of Ag-d states. The CBM is predominantly composed of Bi-p and I-p (analogous to the Pb-p and I-p makeup for the $\mathrm{Pb}$ system). ${ }^{74}$ The composition of the frontier levels in $[\mathrm{AE} 2 \mathrm{~T}]_{2} \mathrm{AgBiI}_{8}$ is also qualitatively similar to that of the $(\mathrm{BA})_{4} \mathrm{AgBiBr}_{8} \mathrm{DP}^{39}$

Structural and Optical Characterization. Figure 3a shows the PXRD patterns of polycrystalline powders and thin films of $[\mathrm{AE} 2 \mathrm{~T}]_{2} \mathrm{AgBiI}_{8}$ (prepared as described in the $\mathrm{SI}$ ) compared to a reference pattern simulated from the
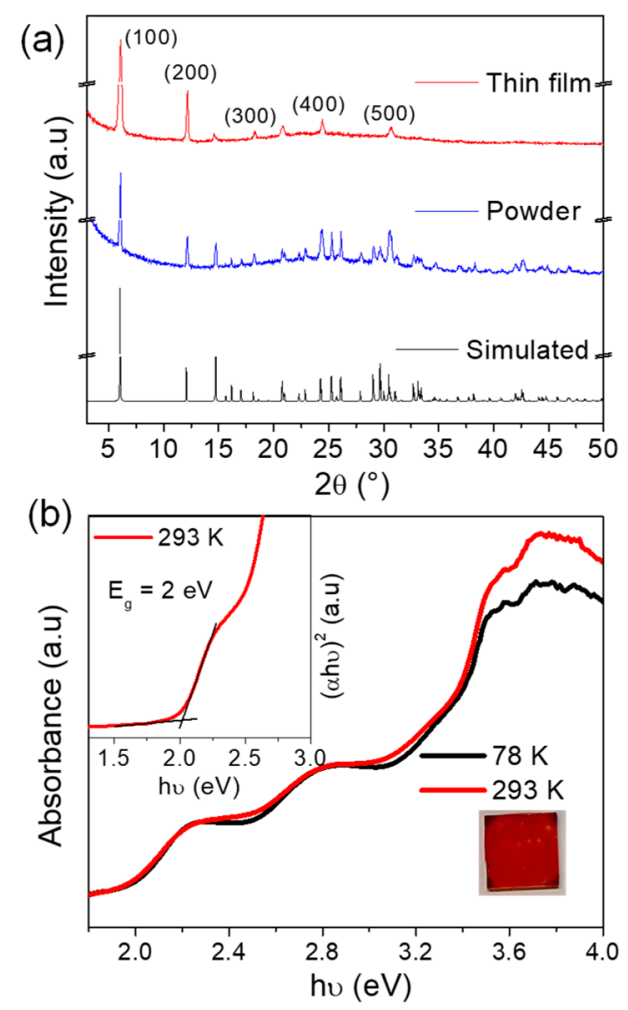

Figure 3. (a) PXRD patterns of thin films and powdered crystals of $[\mathrm{AE} 2 \mathrm{~T}]_{2} \mathrm{AgBiI}_{8}$, along with a simulated pattern (derived from the single-crystal structure) for comparison. (b) Absorption spectra for thin films of $[\mathrm{AE} 2 \mathrm{~T}]_{2} \mathrm{AgBiI}_{8}$ recorded at 78 and $293 \mathrm{~K}$. The upper and lower insets show the corresponding Tauc plot at $293 \mathrm{~K}$ and photograph of the thin film, respectively. experimental room-temperature single-crystal structure. The PXRD patterns agree well with the simulated pattern with no detectable impurities to within the instrumental limit. The absorption spectra of the $[\mathrm{AE} 2 \mathrm{~T}]_{2} \mathrm{AgBiI}_{8}$ film at 293 and $78 \mathrm{~K}$ (Figure $3 \mathrm{~b}$ ) are nearly superimposable, with a slight reduction in the absorbance at energies greater than $3.6 \mathrm{eV}$. The lowestenergy absorption peak is a broad feature centered around 2.3 $\mathrm{eV}$, in contrast to the sharp excitonic peak observed for the analogous Pb-based HOIP. ${ }^{17,42}$ From the corresponding Tauc plot (at $293 \mathrm{~K}$ ), shown in Figure 3b, we find a direct bandgap of $2.00(2) \mathrm{eV}$ for $[\mathrm{AE} 2 \mathrm{~T}]_{2} \mathrm{AgBiI}_{8}$. The exceptional agreement with the theoretical bandgap of $2.01 \mathrm{eV}$ is likely fortuitous in view of the remaining uncertainty from both the computational SOC treatment and the HSE06 density functional, as described above. A direct bandgap was also observed for the $2 \mathrm{D}$ (BA $)_{4} \mathrm{AgBiBr}_{8} \mathrm{DP}$, but with a higher value $(2.85 \mathrm{eV})^{39}$ that, as mentioned above, can mostly be attributed to the lighter halide anion. Similar to our findings, the lowest-energy absorption feature appears broad for $(\mathrm{BA})_{4} \mathrm{AgBiBr}_{8}$, as compared to the narrow excitonic peaks observed in $2 \mathrm{D}$ lead halide perovskites. While it would be tempting to interpret the lowest-energy absorption peak in $[\mathrm{AE} 2 \mathrm{~T}]_{2} \mathrm{AgBiI}_{8}$ as a broadened excitonic feature, this interpretation is not supported by its very weak temperature dependence (Figure $3 b$ ), as well as by the absence of corresponding PL (see below); also in line with the interpretation of the equivalent peak for $(\mathrm{BA})_{4} \mathrm{AgBiBr}_{8} .{ }^{39}$

Interestingly, the computed absorption spectra for $[\mathrm{AE} 2 \mathrm{~T}]_{2}$ $\mathrm{AgBiI}_{8}$ in Figure $\mathrm{S} 11$ are remarkably similar to the experimentally determined curves. The spectra in Figure S11 are calculated at the DFT-HSE06+SOC level of theory and employing the independent-particle approximation, using the same implementation as in ref 65 and neglecting any excitonic effects. Following this approximation, the first absorption peak would be related to the inorganic components at the onsets of the VBM and CBM, since there is no discernible partial contribution of the organic moiety to the first DOS peak that defines the CBM in Figure $2 b$ and $c$. The inorganic DOS contributions are further decomposed in Figure $4 a-f$, revealing the inorganic VBM to be derived from Ag-d and I-p orbitals, whereas the CBM originates from Bi-p and I-p (Figure 4a and b). A similar decomposition for a Br-based DP was performed in ref 39, where the analogous near-band-edge absorption was associated with "considerable $\mathrm{Ag}$ to $\mathrm{Bi}$ charge-transfer (CT) character". In line with an analysis of $\mathrm{Au}(\mathrm{I})-\mathrm{Au}(\mathrm{III}) \mathrm{DP},{ }^{41}$ the actual absorption in $[\mathrm{AE} 2 \mathrm{~T}]_{2} \mathrm{AgBiI}_{8}$ is expected to be mediated by the halide ion connecting Ag and $\mathrm{Bi}$. The partial DOS in Figure $4 \mathrm{a}$ and $\mathrm{b}$ shows that a direct metal-to-metal CT cannot be responsible for the spectra computed in Figure S11; that is, the Ag- $\mathrm{d}$ component (VBM) is zero in the CBM, and vice versa for Bi-p in the CBM, leaving little room for a nonzero metal-tometal transition dipole matrix element.

A closer analysis of the I-p DOS (Figure 4c,d) reveals considerable contributions of the equatorially bonded I atoms $\left(\mathrm{I}_{\text {eq }}\right)$, which connect $\mathrm{Ag}$ and $\mathrm{Bi}$ atoms, to both the VBM and CBM. The observed absorption near the band edges is therefore consistent with transitions between $\mathrm{I}_{\mathrm{eq}}-\mathrm{p}$ states in the VBM and hybridized $I_{e q}-p$ and Bi-p states in the conduction band. Finally, the axially bonded I atoms, $\mathrm{I}_{\mathrm{ax}}$, cannot contribute to near-band-edge absorption; their PDOS (Figures 4e,f) shows that $I_{a x}$ atoms bonded to Ag contribute to the VBM, but not the CBM, and vice versa for $\mathrm{I}_{\mathrm{ax}}$ atoms bonded to $\mathrm{Bi}$. This is somewhat in contrast to the conventional picture in $\mathrm{Pb}$-based HOIPs, where the VBM derives from an antibonding 


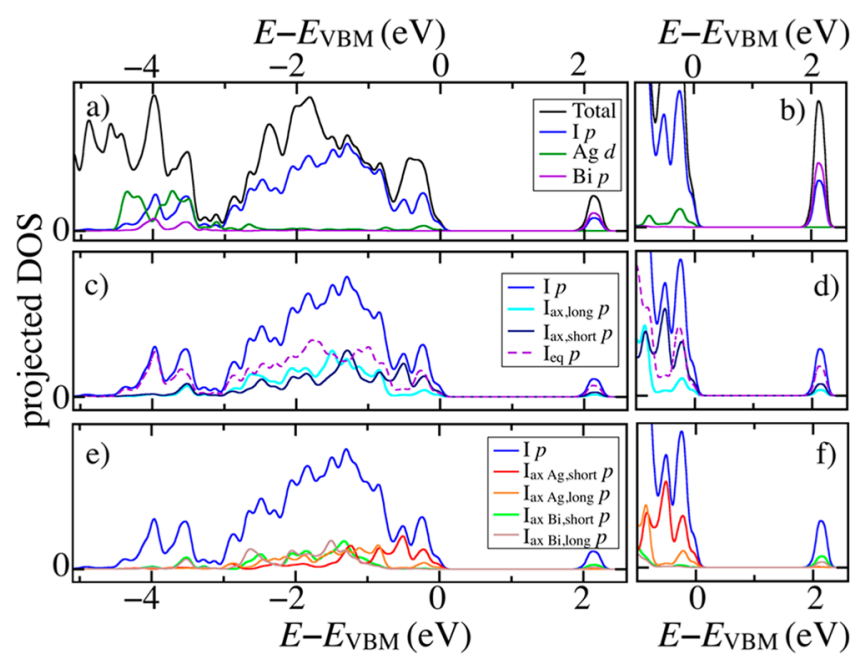

Figure 4. DFT-HSE06+SOC DOS for $[\mathrm{AE} 2 \mathrm{~T}]_{2} \mathrm{AgBiI}_{8}$. (a) Decomposition of the total DOS (black) into I-p (blue), Ag-d (green), and Bi-p (purple) orbital contributions (an enlarged version is shown in Figure 2c). (b) Magnification of band edges from (a). (c) Further separation of the I-p contribution according to I atoms bonded axially to the metal atoms with short $\left(\mathrm{I}_{\mathrm{ax}, \text { shory }}\right.$ dark blue $)$ and long bonds ( $\mathrm{I}_{\mathrm{ax}, \mathrm{long}}$ light blue), as well as equatorial $\mathrm{I}$ atoms ( $\mathrm{I}_{\mathrm{eq}}$, dashed purple) that connect $\mathrm{Ag}$ and $\mathrm{Bi}$ within the inorganic sheets. (d) Magnification of the band edges from (c). (e) I-p contribution to DOS from axially bound I, projected onto Ag-bound axial I with short ( $\mathrm{I}_{\mathrm{ax}, \mathrm{Ag} \text {, short }}$ red) and long ( $\mathrm{I}_{\mathrm{ax}} \mathrm{Ag}$, long, orange) bonds and onto Bibound axial I with short $\left(\mathrm{I}_{\mathrm{ax} \text { Bi, short }}\right.$ green $)$ and long $\left(\mathrm{I}_{\mathrm{ax} B \mathrm{Bi}}\right.$ long, brown $)$ bonds. (f) Magnification of the band edges from (e).

combination of $\mathrm{Pb}-6 \mathrm{~s}$ and I-5p states, while the CBM mainly exhibits contributions from $\mathrm{Pb}-6 \mathrm{p}$ and small contributions from equatorial I-s and axial I-p states. ${ }^{74}$ This band structure is thought to allow for a CT-like direct $\mathrm{p}-\mathrm{p}$ transition within the same metal (i.e., $\mathrm{Pb}) .^{25,39,75}$

Despite its predicted direct bandgap, we find that $[\mathrm{AE} 2 \mathrm{~T}]_{2} \mathrm{AgBiI}_{8}$ is barely luminescent at room temperature and even at $78 \mathrm{~K}$. In contrast, Pb-based HOIPs exhibit a strong and sharp excitonic emission near room temperature. ${ }^{17,42}$ Given the band structure and partial DOS in Figure 2, one possible explanation for quenching could be type IIb band alignment, $^{18}$ i.e., separate VBM (organic) and CBM (inorganic), a scenario that is well within the accuracy limits of current theory. However, the lack of PL is also consistent with the case for $(\mathrm{BA})_{4} \mathrm{AgBiBr}_{8},{ }^{39}$ wherein type II alignment seems much less likely and yet PL is completely quenched near room temperature (a broad and weak defect-mediated emission appears only below $80 \mathrm{~K}$ ). A tentative explanation for the absence of PL is suggested by the character of VBM $\left(\mathrm{I}_{\mathrm{eq}}\right.$ and Ag derived) and CBM ( $\mathrm{I}_{\mathrm{eq}}$ and Bi derived) in Figure 4, indicating that the photogenerated holes would be primarily located on Ag-containing octahedra, whereas the electrons would be located primarily on Bi-containing octahedra. In this scenario, the optical excitations would survive for a long time due to lack of radiative recombination. Indeed, our transient absorption spectroscopy measurements on $[\mathrm{AE} 2 \mathrm{~T}]_{2} \mathrm{AgBiI}_{8}$ thin films reveal a decay of photobleaching through a multistep relaxation process (Figure S12). Within the first few picoseconds, the transient bleaching signal decays but does not completely vanish. After a fast relaxation in the first few picoseconds, the bleaching signal survives longer than $6 \mathrm{~ns}$ (beyond the experimental time window), suggesting the initial transfer of excitations into long-lived dark states.

\section{CONCLUSIONS}

In conclusion, we demonstrate that aromatic interactions in specifically designed bifunctional AE2T spacer cations can template unprecedented 2D DP layers of $\mathrm{Ag}-\mathrm{Bi}$ iodide, otherwise inaccessible with more traditional (i.e., less rigid and lacking substantial aromatic-aromatic interaction) spacer cations. Transition from bromide to iodide leads to a reduction in bandgap to $2.00(2) \mathrm{eV}$, the smallest value reported to date for $2 \mathrm{D}$ DPs. However, in agreement with a previous observation for the $2 \mathrm{D} \mathrm{Ag}-\mathrm{Bi} \mathrm{DP}$ bromide, ${ }^{39}$ we show that replacing $\mathrm{Pb}$ by $\mathrm{Ag}-\mathrm{Bi}$ leads to flatter electronic bands, a relative lack of exciton features, and strongly quenched PL (at least for temperatures $>80 \mathrm{~K}$ ). We determine that the broad, lowest-energy direct transition in $[\mathrm{AE} 2 \mathrm{~T}]_{2} \mathrm{AgBiI}_{8}$ occurs between nominally inorganic-derived states at band extrema and propose that the lack of considerable band-edge PL likely derives from spatial localization/separation of photoexcited holes and electrons in the vicinity of $\mathrm{Ag}$ and $\mathrm{Bi}$, respectively. $[\mathrm{AE} 2 \mathrm{~T}]_{2} \mathrm{AgBiI}_{8}$ also differs from most other related $2 \mathrm{D}$ HOIPs with traditional spacer cations by the proximity of organic AE2T frontier orbitals to the inorganic VBM and CBM and the formation of a borderline-type Ib/type IIb internal quantum well structure. Our present work emphasizes that specifically designed organic cations can perform a double role within 2D perovskites: stabilizing desirable but otherwise unobtainable inorganic frameworks and simultaneously tuning the nature of frontier orbitals to afford specific optoelectronic character. This latter point has been explored recently for the $\mathrm{Pb}$-based systems $^{18}$ and will be pursued for the $\mathrm{Ag} / \mathrm{Bi}$ analogues in future work.

\section{ASSOCIATED CONTENT}

\section{S Supporting Information}

The Supporting Information is available free of charge on the ACS Publications website at DOI: 10.1021/jacs.9b02909.

Synthesis details of AE2T.2HI salt; experimental and computational methods; 298 and $100 \mathrm{~K}$ single-crystal structural data for $[\mathrm{AE} 2 \mathrm{~T}]_{2} \mathrm{AgBiI}_{8} ; \mathrm{XPS}$ and FESEM characterization of $[\mathrm{AE} 2 \mathrm{~T}]_{2} \mathrm{AgBiI}_{8}$ single crystals; details of $[\mathrm{AE} 2 \mathrm{~T}]_{2} \mathrm{AgBiI}_{8}$ theoretical structure; comparison of experimental and theoretical lattice constants, bond lengths, and bond angles; calculated reference band structures and DOS for $\mathrm{Cs}_{2} \mathrm{AgBiCl}_{6}$ and calculated reference band structure for $[\mathrm{AE} 2 \mathrm{~T}] \mathrm{PbI}_{4}$; calculated absorption coefficient; and transient absorption spectra along with corresponding decay kinetics for [AE2$\mathrm{T}]_{2} \mathrm{AgBiI}_{8}$ thin film (PDF)

Crystallographic data for $[\mathrm{AE} 2 \mathrm{~T}]_{2} \mathrm{AgBiI}_{8} 100 \mathrm{~K}$ (CIF) Crystallographic data for $[\mathrm{AE} 2 \mathrm{~T}]_{2} \mathrm{AgBiI}_{8} 298 \mathrm{~K}$ (CIF)

\section{AUTHOR INFORMATION}

\section{Corresponding Author}

*david.mitzi@duke.edu

ORCID $\odot$

Volker Blum: 0000-0001-8660-7230

David B. Mitzi: 0000-0001-5189-4612

Notes

The authors declare no competing financial interest. 


\section{ACKNOWLEDGMENTS}

M.K.J., D.B.M., S.M.J., V.B., C.L., D.D., W.Y., S.D., and K.G. acknowledge support from the NSF DMREF program $\left(\mathrm{HybriD}^{3}\right)$ under grant numbers DMR-1729297, DMR1728921, and DMR-1729383. An award of computer time was provided by the INCITE program. This research used resources of the Argonne Leadership Computing Facility, which is a DOE Office of Science User Facility supported under Contract DE-AC02-06CH11357. S.M.J. thanks the Deutsche Forschungsgemeinschaft (DFG, German Research Foundation) for a postdoctoral fellowship, grant number 393196393.

\section{REFERENCES}

(1) Mitzi, D. B.; Dimitrakopoulos, C. D.; Rosner, J.; Medeiros, D. R.; Xu, Z.; Noyan, C. Hybrid Field-Effect Transistor Based on a LowTemperature Melt-Processed Channel Layer. Adv. Mater. 2002, 14, 1772 .

(2) Kagan, C. R.; Mitzi, D. B.; Dimitrakopoulos, C. D. OrganicInorganic Hybrid Materials as Semiconducting Channels in Thin-Film Field-Effect Transistors. Science 1999, 286, 945.

(3) Van Le, Q.; Jang, H. W.; Kim, S. Y. Recent Advances toward High-Efficiency Halide Perovskite Light-Emitting Diodes: Review and Perspective. Small Methods. 2018, 2, 1700419.

(4) Zhao, B.; Bai, S.; Kim, V.; Lamboll, R.; Shivanna, R.; Auras, F.; Richter, J. M.; Yang, L.; Dai, L.; Alsari, M.; She, X.-J.; Liang, L.; Zhang, J.; Lilliu, S.; Gao, P.; Snaith, H. J.; Wang, J.; Greenham, N. C.; Friend, R. H.; Di, D. High-efficiency perovskite-polymer bulk heterostructure light-emitting diodes. Nat. Photonics 2018, 12, 783.

(5) Zhao, X.; Ng, J. D. A.; Friend, R. H.; Tan, Z.-K. Opportunities and Challenges in Perovskite Light-Emitting Devices. ACS Photonics 2018, 5, 3866

(6) Tsai, H.; Nie, W.; Blancon, J.-C.; Stoumpos, C. C.; Asadpour, R.; Harutyunyan, B.; Neukirch, A. J.; Verduzco, R.; Crochet, J. J.; Tretiak, S.; Pedesseau, L.; Even, J.; Alam, M. A.; Gupta, G.; Lou, J.; Ajayan, P. M.; Bedzyk, M. J.; Kanatzidis, M. G.; Mohite, A. D. High-efficiency two-dimensional Ruddlesden-Popper perovskite solar cells. Nature 2016, 536, 312.

(7) Smith, I. C.; Hoke, E. T.; Solis-Ibarra, D.; McGehee, M. D.; Karunadasa, H. I. A Layered Hybrid Perovskite Solar-Cell Absorber with Enhanced Moisture Stability. Angew. Chem. 2014, 126, 11414.

(8) Cao, D. H.; Stoumpos, C. C.; Farha, O. K.; Hupp, J. T.; Kanatzidis, M. G. 2D Homologous Perovskites as Light-Absorbing Materials for Solar Cell Applications. J. Am. Chem. Soc. 2015, 137, 7843.

(9) Mao, L.; Ke, W.; Pedesseau, L.; Wu, Y.; Katan, C.; Even, J.; Wasielewski, M. R.; Stoumpos, C. C.; Kanatzidis, M. G. Hybrid DionJacobson 2D Lead Iodide Perovskites. J. Am. Chem. Soc. 2018, 140, 3775.

(10) Li, Y.; Milić, J. V.; Ummadisingu, A.; Seo, J.-Y.; Im, J.-H.; Kim, H.-S.; Liu, Y.; Dar, M. I.; Zakeeruddin, S. M.; Wang, P.; Hagfeldt, A.; Grätzel, M. Bifunctional Organic Spacers for Formamidinium-Based Hybrid Dion-Jacobson Two-Dimensional Perovskite Solar Cells. Nano Lett. 2019, 19, 150.

(11) Yin, J.; Maity, P.; Xu, L.; El-Zohry, A. M.; Li, H.; Bakr, O. M.; Brédas, J.-L.; Mohammed, O. F. Layer-Dependent Rashba Band Splitting in 2D Hybrid Perovskites. Chem. Mater. 2018, 30, 8538.

(12) Zhai, Y.; Baniya, S.; Zhang, C.; Li, J.; Haney, P.; Sheng, C.-X.; Ehrenfreund, E.; Vardeny, Z. V. Giant Rashba splitting in 2D organicinorganic halide perovskites measured by transient spectroscopies. Science Adv. 2017, 3, No. e1700704.

(13) Saparov, B.; Mitzi, D. B. Organic-Inorganic Perovskites: Structural Versatility for Functional Materials Design. Chem. Rev. 2016, 116, 4558.

(14) Mao, L.; Stoumpos, C. C.; Kanatzidis, M. G. Two-Dimensional Hybrid Halide Perovskites: Principles and Promises. J. Am. Chem. Soc. 2019, 141, 1171.
(15) Mitzi, D. B.; Chondroudis, K.; Kagan, C. R. Organic-inorganic electronics. IBM J. Res. Dev. 2001, 45, 29.

(16) Hong, X.; Ishihara, T.; Nurmikko, A. V. Photoconductivity and electroluminescence in lead iodide based natural quantum well structures. Solid State Commun. 1992, 84, 657.

(17) Mitzi, D. B.; Chondroudis, K.; Kagan, C. R. Design, Structure, and Optical Properties of Organic-Inorganic Perovskites Containing an Oligothiophene Chromophore. Inorg. Chem. 1999, 38, 6246.

(18) Liu, C.; Huhn, W.; Du, K.-Z.; Vazquez-Mayagoitia, A.; Dirkes, D.; You, W.; Kanai, Y.; Mitzi, D. B.; Blum, V. Tunable Semiconductors: Control over Carrier States and Excitations in Layered Hybrid Organic-Inorganic Perovskites. Phys. Rev. Lett. 2018, 121, 146401.

(19) Ema, K.; Inomata, M.; Kato, Y.; Kunugita, H.; Era, M. Nearly Perfect Triplet-Triplet Energy Transfer from Wannier Excitons to Naphthalene in Organic-Inorganic Hybrid Quantum-Well Materials. Phys. Rev. Lett. 2008, 100, 257401.

(20) Hu, H.; Meier, F.; Zhao, D.; Abe, Y.; Gao, Y.; Chen, B.; Salim, T.; Chia, E. E. M.; Qiao, X.; Deibel, C.; Lam, Y. M. Efficient RoomTemperature Phosphorescence from Organic-Inorganic Hybrid Perovskites by Molecular Engineering. Adv. Mater. 2018, 30, 1707621.

(21) Passarelli, J. V.; Fairfield, D. J.; Sather, N. A.; Hendricks, M. P.; Sai, H.; Stern, C. L.; Stupp, S. I. Enhanced Out-of-Plane Conductivity and Photovoltaic Performance in $\mathrm{n}=1$ Layered Perovskites through Organic Cation Design. J. Am. Chem. Soc. 2018, 140, 7313.

(22) Gao, P.; Bin Mohd Yusoff, A. R.; Nazeeruddin, M. K. Dimensionality engineering of hybrid halide perovskite light absorbers. Nat. Commun. 2018, 9, 5028.

(23) Zhang, S.-F.; Chen, X.-K.; Ren, A.-M.; Li, H.; Bredas, J.-L. Impact of Organic Spacers on the Carrier Dynamics in 2D Hybrid Lead-Halide Perovskites. ACS Energy Lett. 2019, 4, 17.

(24) Maheshwari, S.; Savenije, T. J.; Renaud, N.; Grozema, F. C. Computational Design of Two-Dimensional Perovskites with Functional Organic Cations. J. Phys. Chem. C 2018, 122, 17118.

(25) Yin, W.-J.; Shi, T.; Yan, Y. Unique Properties of Halide Perovskites as Possible Origins of the Superior Solar Cell Performance. Adv. Mater. 2014, 26, 4653.

(26) Fisher, G. A.; Norman, N. C. In Advances in Inorganic Chemistry; Sykes, A. G., Ed.; Academic Press, 1994; Vol. 41, p 233.

(27) Pohl, S.; Lotz, R.; Saak, W.; Haase, D. Structural Diversity in Iodoantimonates; the Anions $\mathrm{Sb}_{3} \mathrm{I}, \mathrm{Sb}_{5} \mathrm{I}$ and $\mathrm{Sb}_{6} \mathrm{I}$. Angew. Chem., Int. Ed. Engl. 1989, 28, 344.

(28) Lindsjö, M.; Fischer, A.; Kloo, L. Anionic Diversity in Iodobismuthate Chemistry. Z. Anorg. Allg. Chem. 2005, 631, 1497.

(29) Goforth, A. M.; Tershansy, M. A.; Smith, M. D.; Peterson, L.; Kelley, J. G.; DeBenedetti, W. J. I.; zur Loye, H.-C. Structural Diversity and Thermochromic Properties of Iodobismuthate Materials Containing d-Metal Coordination Cations: Observation of a High Symmetry $\left[\mathrm{Bi}_{3} \mathrm{I}_{11}\right]^{2-}$ Anion and of Isolated $\mathrm{I}^{-}$Anions. J. Am. Chem. Soc. 2011, 133, 603.

(30) Volonakis, G.; Haghighirad, A. A.; Milot, R. L.; Sio, W. H.; Filip, M. R.; Wenger, B.; Johnston, M. B.; Herz, L. M.; Snaith, H. J.; Giustino, F. $\mathrm{Cs}_{2} \mathrm{InAgCl}_{6}$ : A New Lead-Free Halide Double Perovskite with Direct Band Gap. J. Phys. Chem. Lett. 2017, 8, 772.

(31) Du, K.-Z.; Meng, W.; Wang, X.; Yan, Y.; Mitzi, D. B. Bandgap Engineering of Lead-Free Double Perovskite $\mathrm{Cs}_{2} \mathrm{AgBiBr}_{6}$ through Trivalent Metal Alloying. Angew. Chem., Int. Ed. 2017, 56, 8158.

(32) McClure, E. T.; Ball, M. R.; Windl, W.; Woodward, P. M. $\mathrm{Cs}_{2} \mathrm{AgBiX}_{6}(\mathrm{X}=\mathrm{Br}, \mathrm{Cl})$ : New Visible Light Absorbing, Lead-Free Halide Perovskite Semiconductors. Chem. Mater. 2016, 28, 1348.

(33) Pantaler, M.; Cho, K. T.; Queloz, V. I. E.; García Benito, I.; Fettkenhauer, C.; Anusca, I.; Nazeeruddin, M. K.; Lupascu, D. C.; Grancini, G. Hysteresis-Free Lead-Free Double-Perovskite Solar Cells by Interface Engineering. ACS Energy Lett. 2018, 3, 1781.

(34) Slavney, A. H.; Leppert, L.; Saldivar-Valdes, A.; Bartesaghi, D.; Savenije, T. J.; Neaton, J. B.; Karunadasa, H. I. Small-Band-Gap Halide Double Perovskites. Angew. Chem., Int. Ed. 2018, 57, 12765. 
(35) Vargas, B.; Ramos, E.; Pérez-Gutiérrez, E.; Alonso, J. C.; SolisIbarra, D. A Direct Bandgap Copper-Antimony Halide Perovskite. J. Am. Chem. Soc. 2017, 139, 9116.

(36) Zhao, X.-G.; Yang, D.; Ren, J.-C.; Sun, Y.; Xiao, Z.; Zhang, L. Rational Design of Halide Double Perovskites for Optoelectronic Applications. Joule. 2018, 2, 1662.

(37) Xiao, Z.; Du, K.-Z.; Meng, W.; Wang, J.; Mitzi, D. B.; Yan, Y. Intrinsic Instability of $\mathrm{Cs}_{2} \mathrm{In}(\mathrm{I}) \mathrm{M}(\mathrm{III}) \mathrm{X}_{6}(\mathrm{M}=\mathrm{Bi}, \mathrm{Sb} ; \mathrm{X}=$ Halogen $)$ Double Perovskites: A Combined Density Functional Theory and Experimental Study. J. Am. Chem. Soc. 2017, 139, 6054.

(38) Creutz, S. E.; Crites, E. N.; De Siena, M. C.; Gamelin, D. R. Colloidal Nanocrystals of Lead-Free Double-Perovskite (Elpasolite) Semiconductors: Synthesis and Anion Exchange To Access New Materials. Nano Lett. 2018, 18, 1118.

(39) Connor, B. A.; Leppert, L.; Smith, M. D.; Neaton, J. B.; Karunadasa, H. I. Layered Halide Double Perovskites: Dimensional Reduction of $\mathrm{Cs}_{2} \mathrm{AgBiBr}_{6}$. J. Am. Chem. Soc. 2018, 140, 5235.

(40) Castro-Castro, L. M.; Guloy, A. M. Organic-Based Layered Perovskites of Mixed-Valent Gold(I)/Gold(III) Iodides. Angew. Chem., Int. Ed. 2003, 42, 2771.

(41) Kojima, N.; Kitagawa, H. Optical investigation of the intervalence charge-transfer interactions in the three-dimensional gold mixed-valence compounds $\mathrm{Cs}_{2} \mathrm{Au}_{2} \mathrm{X}_{6}(\mathrm{X}=\mathrm{Cl}, \mathrm{Br}$ or I $)$. J. Chem. Soc., Dalton Trans. 1994, 327.

(42) Blancon, J. C.; Stier, A. V.; Tsai, H.; Nie, W.; Stoumpos, C. C.; Traoré, B.; Pedesseau, L.; Kepenekian, M.; Katsutani, F.; Noe, G. T.; Kono, J.; Tretiak, S.; Crooker, S. A.; Katan, C.; Kanatzidis, M. G.; Crochet, J. J.; Even, J.; Mohite, A. D. Scaling law for excitons in 2D perovskite quantum wells. Nat. Commun. 2018, 9, 2254.

(43) Mitzi, D. B.; Dimitrakopoulos, C. D.; Kosbar, L. L. Structurally Tailored Organic-Inorganic Perovskites: Optical Properties and Solution-Processed Channel Materials for Thin-Film Transistors. Chem. Mater. 2001, 13, 3728.

(44) Mitzi, D. B. Organic-Inorganic Perovskites Containing Trivalent Metal Halide Layers: The Templating Influence of the Organic Cation Layer. Inorg. Chem. 2000, 39, 6107.

(45) Chaloner, P. A.; Gunatunga, S. R.; Hitchcock, P. B. Redetermination of 2,2'-bithiophene. Acta Crystallogr., Sect. C: Cryst. Struct. Commun. 1994, 50, 1941.

(46) Zhu, X.-H.; Mercier, N.; Frère, P.; Blanchard, P.; Roncali, J.; Allain, M.; Pasquier, C.; Riou, A. Effect of Mono- versus Diammonium Cation of 2,2'-Bithiophene Derivatives on the Structure of Organic-Inorganic Hybrid Materials Based on Iodo Metallates. Inorg. Chem. 2003, 42, 5330.

(47) Mitzi, D. B.; Brock, P. Structure and Optical Properties of Several Organic-Inorganic Hybrids Containing Corner-Sharing Chains of Bismuth Iodide Octahedra. Inorg. Chem. 2001, 40, 2096.

(48) Jorgensen, W. L.; Severance, D. L. Aromatic-aromatic interactions: free energy profiles for the benzene dimer in water, chloroform, and liquid benzene. J. Am. Chem. Soc. 1990, 112, 4768.

(49) Oldag, T.; Aussieker, T.; Keller, H.-L.; Preitschaft, C.; Pfitzner, A. Solvothermal Synthesis and Crystal Structure Determination of $\mathrm{AgBiI}_{4}$ and $\mathrm{Ag}_{3} \mathrm{BiI}_{6}$. Z. Anorg. Allg. Chem. 2005, 631, 677.

(50) Blum, V.; Gehrke, R.; Hanke, F.; Havu, P.; Havu, V.; Ren, X.; Reuter, K.; Scheffler, M. Ab initio molecular simulations with numeric atom-centered orbitals. Comput. Phys. Commun. 2009, 180, 2175.

(51) Havu, V.; Blum, V.; Havu, P.; Scheffler, M. Efficient O(N) integration for all-electron electronic structure calculation using numeric basis functions. J. Comput. Phys. 2009, 228, 8367.

(52) Xinguo, R.; Patrick, R.; Volker, B.; Jürgen, W.; Alexandre, T.; Andrea, S.; Karsten, R.; Matthias, S. Resolution-of-identity approach to Hartree-Fock, hybrid density functionals, RPA, MP2 and GW with numeric atom-centered orbital basis functions. New J. Phys. 2012, 14, 053020 .

(53) Levchenko, S. V.; Ren, X.; Wieferink, J.; Johanni, R.; Rinke, P.; Blum, V.; Scheffler, M. Hybrid functionals for large periodic systems in an all-electron, numeric atom-centered basis framework. Comput. Phys. Commun. 2015, 192, 60.
(54) Knuth, F.; Carbogno, C.; Atalla, V.; Blum, V.; Scheffler, M. Allelectron formalism for total energy strain derivatives and stress tensor components for numeric atom-centered orbitals. Comput. Phys. Commun. 2015, 190, 33.

(55) Perdew, J. P.; Burke, K.; Ernzerhof, M. Generalized Gradient Approximation Made Simple. Phys. Rev. Lett. 1996, 77, 3865.

(56) Tkatchenko, A.; Scheffler, M. Accurate Molecular Van Der Waals Interactions from Ground-State Electron Density and FreeAtom Reference Data. Phys. Rev. Lett. 2009, 102, 073005.

(57) Heyd, J.; Scuseria, G. E.; Ernzerhof, M. Hybrid functionals based on a screened Coulomb potential. J. Chem. Phys. 2003, 118, 8207.

(58) Heyd, J.; Scuseria, G. E.; Ernzerhof, M. Erratum: "Hybrid functionals based on a screened Coulomb potential" [J. Chem. Phys. 118, 8207 (2003)]. J. Chem. Phys. 2006, 124, 219906.

(59) Krukau, A. V.; Vydrov, O. A.; Izmaylov, A. F.; Scuseria, G. E. Influence of the exchange screening parameter on the performance of screened hybrid functionals. J. Chem. Phys. 2006, 125, 224106.

(60) Huhn, W. P.; Blum, V. One-hundred-three compound bandstructure benchmark of post-self-consistent spin-orbit coupling treatments in density functional theory. Phys. Rev. Materials. 2017, $1,033803$.

(61) Li, T.; Zhao, X.; Yang, D.; Du, M.-H.; Zhang, L. Intrinsic Defect Properties in Halide Double Perovskites for Optoelectronic Applications. Phys. Rev. Appl. 2018, 10, 041001.

(62) Blaha, P.; Schwarz, K.; Madsen, G. K. H.; Kvasnicka, D.; Luitz, J. WIEN2K, An Augmented Plane Wave + Local Orbitals Program for Calculating Crystal Properties; Karlheinz Schwarz, Techn. Universität Wien, Austria: Wien, Austria, 2001.

(63) Paier, J.; Asahi, R.; Nagoya, A.; Kresse, G. $\mathrm{Cu}_{2} \mathrm{ZnSnS}_{4}$ as a potential photovoltaic material: A hybrid Hartree-Fock density functional theory study. Phys. Rev. B: Condens. Matter Mater. Phys. 2009, 79, 115126.

(64) Skone, J. H.; Govoni, M.; Galli, G. Nonempirical rangeseparated hybrid functionals for solids and molecules. Phys. Rev. B: Condens. Matter Mater. Phys. 2016, 93, 235106.

(65) Zhu, T.; Huhn, W. P.; Wessler, G. C.; Shin, D.; Saparov, B.; Mitzi, D. B.; Blum, V. I-II-IV-VI $4(\mathrm{I}=\mathrm{Cu}, \mathrm{Ag}$; II = Sr, Ba; IV = Ge, $\mathrm{Sn} ; \mathrm{VI}=\mathrm{S}, \mathrm{Se})$ : Chalcogenides for Thin-Film Photovoltaics. Chem. Mater. 2017, 29, 7868.

(66) Kim, J.; Kim, H.; Chandran, M.; Lee, S.-C.; Im, S. H.; Hong, K.-H. Impacts of cation ordering on bandgap dispersion of double perovskites. APL Mater. 2018, 6, 084903.

(67) Savory, C. N.; Walsh, A.; Scanlon, D. O. Can Pb-Free Halide Double Perovskites Support High-Efficiency Solar Cells? ACS Energy Lett. 2016, 1, 949.

(68) Yang, J.; Zhang, P.; Wei, S.-H. Band Structure Engineering of $\mathrm{Cs}_{2} \mathrm{AgBiBr}_{6}$ Perovskite through Order-Disordered Transition: A FirstPrinciple Study. J. Phys. Chem. Lett. 2018, 9, 31.

(69) Hoyer, M.; Hartl, H. Die Kristallstrukturen von $\mathrm{Tl}_{2} \mathrm{AgI}_{3}$ und $\mathrm{NaAgI}_{2} \cdot 3 \mathrm{H}_{2} \mathrm{O} . \mathrm{Z}$. Anorg. Allg. Chem. 1996, 622, 308.

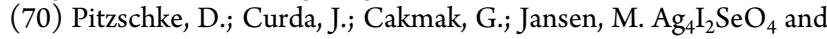
$\mathrm{Ag}_{3} \mathrm{ITeO}_{4}$ - Two New Silver Solid Electrolytes. Z. Anorg. Allg. Chem. 2008, 634, 1071.

(71) Filip, M. R.; Hillman, S.; Haghighirad, A. A.; Snaith, H. J.; Giustino, F. Band Gaps of the Lead-Free Halide Double Perovskites $\mathrm{Cs}_{2} \mathrm{BiAgCl}_{6}$ and $\mathrm{Cs}_{2} \mathrm{BiAgBr}_{6}$ from Theory and Experiment. J. Phys. Chem. Lett. 2016, 7, 2579.

(72) Even, J.; Pedesseau, L.; Jancu, J.-M.; Katan, C. Importance of Spin-Orbit Coupling in Hybrid Organic/Inorganic Perovskites for Photovoltaic Applications. J. Phys. Chem. Lett. 2013, 4, 2999.

(73) Even, J.; Pedesseau, L.; Dupertuis, M. A.; Jancu, J. M.; Katan, C. Electronic model for self-assembled hybrid organic/perovskite semiconductors: Reverse band edge electronic states ordering and spin-orbit coupling. Phys. Rev. B: Condens. Matter Mater. Phys. 2012, 86,205301

(74) Katan, C.; Mercier, N.; Even, J. Quantum and Dielectric Confinement Effects in Lower-Dimensional Hybrid Perovskite Semiconductors. Chem. Rev. 2019, 119, 3140. 
Journal of the American Chemical Society

(75) Ishihara, T.; Hirasawa, M.; Goto, T. Optical Properties and Electronic Structures of Self-Organized Quantum Well $\left(\mathrm{C}_{\mathrm{n}} \mathrm{H}_{2 \mathrm{n}+1} \mathrm{NH}_{3}\right)_{2} \mathrm{PbX}{ }_{4}(\mathrm{X}=\mathrm{I}, \mathrm{Br}, \mathrm{Cl})$. Jpn. J. Appl. Phys. 1995, 34, 71. 